\title{
Learning what to expect: context-specific control over intertrial priming effects in singleton search
}

\author{
David R. Thomson • Michael D’Ascenzo • Bruce Milliken
}

Published online: 22 December 2012

(C) Psychonomic Society, Inc. 2012

\begin{abstract}
The present study explored the degree to which repetition effects in color pop-out search from trial $n-1$ to trial $n$ are subject to the attentional control settings of the observer. Intertrial priming effects were compared between two contexts that differed in terms of the utility of immediate prior experience for current performance; in one context, the target was likely to repeat, and in the other context, the target was likely to alternate from one trial to the next. Across two experiments, priming of pop-out (PoP) effects (Malkjovic \& Nakayama; Memory \& Cognition 22:657-672, 1994) were modulated in accord with the probability of target color repetition in a given trial context. Importantly, this modulation persisted when trial history preceding trial $n-1$ was controlled for. Furthermore, this control over PoP seems not to derive from explicit strategies and is not an artifact of randomly occurring strings of same-target trials. We argue that priming effects in singleton search from trial $n-1$ to trial $n$ are subject to a form of implicit top-down control.
\end{abstract}

Keywords Attention $\cdot$ Memory $\cdot$ Visual search

\section{Introduction}

Searching for an item in our perceptual environment that differs from all other items in color, size, motion, or orientation is a tremendously easy task. In fact, it seems as though we do not search for such items at all but, rather, as though the focus of our attention is "grabbed" by them automatically. Nonetheless, although such searches seem effortless, the time taken to complete them can be greatly affected by recent prior experiences.

D. R. Thomson $(\triangle) \cdot$ M. D'Ascenzo $\cdot$ B. Milliken Department of Psychology, Neuroscience \& Behavior, McMaster University, 1280 Main St. West,

Hamilton, ON, Canada L8S 4K1

e-mail: thomsodr@mcmaster.ca
For example, Malkjovic and Nakayama (1994) showed that response times (RTs) in a color pop-out search task (first used by Bravo \& Nakayama, 1992) are significantly shorter when the color of the target on the current trial repeats from the previous trial, relative to when it switches - the now well-known priming of pop-out (PoP) effect. Although there has been much work in recent years devoted to understanding the properties and mechanisms that underlie PoP, whether or not expectation concerning the upcoming target color can affect the magnitude of PoP has yet to be resolved definitively. The present study demonstrates that priming effects in simple pop-out search can indeed be affected by the expectations of the observer.

To explore the effect of expectation on PoP effects, we must first define what we mean by expectation. Although expectation often refers to explicit and conscious knowledge that is used strategically to prepare for a probable future event, in the present work, we use the term more broadly to refer to any sense in which prior experience results in a state of preparedness for a probable future event, whether that preparation is implicit or explicit. In fact, one could easily argue that many forms of expectation are entirely implicit, such as the expectations that the perceptual-motor system generates during the learning of complex sequences (see Nissen \& Bullemer, 1987). There is also precedent in the visual search literature for discussing the effects of knowledge (sometimes referred to as top-down processes) that are implicit in nature. For example, the fact that priming effects are, by definition, influences on behavior that are outside the constraints of current perception has led some researchers to posit that implicit, yet top-down, control mechanisms contribute to (or even drive) priming effects (Geyer \& Muller, 2009; Wolfe, Butcher, Lee, \& Hyle, 2003). We therefore seek to examine the role of expectations on PoP effects, regardless of whether these expectations develop consciously or not.

Although it may appear at face value to be a relatively simple issue, the role of expectation in singleton search is 
not yet clear, since the researchers who have explored this issue have arrived at somewhat different conclusions. The first attempt to manipulate expectations in color pop-out search was made by Malkjovic and Nakayama (1994, Experiment 2). In their experiment, participants searched for an odd-colored diamond that was either red or green on each trial and indicated whether it was chipped on the left or the right side (a compound search task). Three observers performed five blocks of trials in which the probability of receiving a target color switch on the current trial, relative to the immediately preceding trial, was $0, .1, .5, .7, .9$, or 1 . Importantly, they found that mean RTs for each block increased in a somewhat linear manner as the probability of a target switch increased. This result demonstrated that even when observers could expect a target color switch on each trial, RT was significantly longer than when they expected a repetition on each trial. In other words, performance did not unfold in accord with the predictability of the target feature on each trial within a block but, rather, in accord with the number of switch trials within a block. This result led Malkjovic and Nakayama (1994) to conclude that expectation has little or no impact on overall search times for oddcolored targets. However, it is important to note that they did not directly assess how expectation affects trial-to-trial priming effects.

To explore whether expectation modulates trial-to-trial feature repetition effects in pop-out search, Hillstrom (2000, Experiment 1) compared performance in an odd-color search task between a condition in which target colors switched predictably on every other trial (i.e., pink, pink, purple, purple, pink, pink) and a condition in which target colors switched randomly. A simple prediction for this experiment might be that the perfect predictability for both repetitions and alternations in the predictable condition would speed RTs equally for both conditions and, therefore, leave priming effects unchanged, relative to the random condition. However, priming effects were larger when upcoming target colors were predictable, relative to when they were not. This result suggests specifically that the effects of expectation are asymmetrical with respect to repetitions and alternations (having a larger effect on repetitions). More broadly, this result also implies that expectation can indeed impact trial-to-trial priming effects in singleton search, despite the fact that overall RTs seem to increase as the proportion of switch trials increases, as per Malkjovic and Nakayama (1994).

More recently, the effects of expectation on PoP were examined by Geyer and Muller (2009). In their experiments, RTs decreased significantly as the number of consecutive repetitions of a target color increased across trials. This finding had been reported previously by Maljkovic and Nakayama (2000) and is commonly referred to as a cumulative repetition benefit. More important, Geyer and Muller found that this cumulative repetition benefit was larger in an experiment in which the probability of cumulative repetitions was high (e.g., following a switch trial, $p=.90$ for one repetition, .81 for two repetitions, .72 for three repetitions, etc.) than in a baseline experiment with a lower proportion of target color repetitions $(p=.50)$. As a result, it was concluded that observers capitalize on successive repetitions of target color more when successive repetitions are expected. Curiously, however, the proportion of trial-to-trial target color repetitions had no measurable effect on the magnitude of priming observed from trial $n-1$ to trial $n$ when cumulative repetitions ( $>2$ consecutive target color repetitions) were controlled for. This result led these authors to conclude that such first-order priming effects may be invulnerable to top-down control.

The conclusion of Geyer and Muller (2009) that firstorder (i.e., trial $n-1$ to trial $n$ in the absence of successive repetitions) priming effects are not affected by expectation is particularly interesting when contrasted with the results of Hillstrom (2000) reported above. Recall that in her predictable condition, participants alternated between target color repetitions and switches on each trial, and so there was no opportunity for cumulative repetition benefits to accrue. With this in mind, the fact that Hillstrom found larger PoP effects in her predictable condition (in which all repetitions were first-order repetitions) is at odds with Geyer and Muller's conclusion. The focus of the present set of experiments, therefore, was to resolve definitively the issue of whether expectation can modulate trial-to-trial priming effects in color pop-out search.

The experiments that follow manipulated the probability that a target color repetition or alternation occurred on each trial, such that one block of trials was associated with a high probability of repetition and a separate block of trials was associated with a low probability of repetition. Comparing priming effects in a high-proportion-repeated condition with those observed in a low-proportion-repeated condition allows for a much stronger comparison of priming effects than in Geyer and Muller (2009), while at the same time encouraging only one type of expectancy within each block (recall that Hillstrom, 2000, manipulated expectancy for both repetitions and alternations within a single block). In Experiment 1, we show that PoP effects do indeed differ between high- and lowproportion-repeated contexts. This result is to be expected, since cumulative repetition benefits alone would produce much larger PoP effects in a condition with many successive target color repetitions. However, a key result was that differences in first-order repetition effects remained when cumulative repetition effects were controlled for in the analysis. In Experiment 2, we show that PoP effects again differ as a function of our proportion-repeated manipulation, even when prior search trial history is controlled for between the highand low-proportion-repeated contexts; evidence that PoP effects (from trial $n-1$ to trial $n$ ) can be modulated by the statistics of one's past experiences. 


\section{Experiment 1}

One of the purposes of Experiment 1 was to replicate the results obtained by Malkjovic and Nakayama (1994, Experiment 2) that RTs increase as the number of target switch trials within a block increases. Second, we wanted to examine whether PoP effects would be affected by the probability of a target repetition (or switch) within an experimental block. Indeed, given the well-documented presence of cumulative repetition benefits in such tasks, mean target color repeat RTs in a high-proportionrepeated condition will derive from a large number of consecutive target color repetitions, and therefore, we expected that the resulting priming effect should be larger than in a lowproportion-repeated condition, in which successive target color repetitions would be relatively uncommon. Finally, our most important objective was to examine whether priming effects from trial $n-1$ to trial $n$ would be larger in the high- than in the low-proportion-repeated conditions with prior trial history controlled for (so-called first-order effects), a finding that was absent in the Geyer and Muller (2009) study when first-order repetition effects in a high-proportion-repeated condition were compared with those in a baseline condition.

\section{Method}

\section{Participants}

Twenty-four undergraduates at McMaster University (6 male, 18 female; $M_{\text {age }}=18.8$ years) enrolled in introductory psychology took part in exchange for course credit. All participants had normal or corrected-to-normal vision.

\section{Apparatus and stimuli}

Search arrays were presented using Presentation software (Version .070, www.neurobs.com) on a Dell 15-in. CRT monitor connected to a Dell personal computer. Each search array contained three squares that subtended a vertical and horizontal angle of $1.1^{\circ}$. Each square contained a gap in either the left or right side that subtended a visual angle of $0.6^{\circ}$ vertically. Each search array was arranged in an upright equilateral triangle with a fixation cross at the center, which subtended a vertical and horizontal visual angle of $0.6^{\circ}$. Each array contained two homogeneous distractor items and one target item (i.e., if the target item was randomly assigned to be red on a given trial, the distractors were presented in green). Each search array was centered on the computer screen.

\section{Procedure}

Participants were seated approximately $57 \mathrm{~cm}$ from the computer screen and were instructed that, on each trial, they would see three squares arranged around a central fixation cross. They were asked to locate the odd-colored square in each display and to indicate as quickly and accurately as possible whether it had a gap in the left or right side. Participants indicated a left response by hitting the "Z" key with their left index finger and indicated a right response by hitting the "/" key with their right index finger.

Each stimulus array was preceded by a 500-ms blank interval, after which the search array, together with the fixation cross, appeared on the screen. Search arrays remained on the screen until a response was executed. Each experimental session consisted of 1,000 trials and took approximately $40 \mathrm{~min}$ to complete. Participants were given a rest break after every 200 trials and pressed the space bar to resume the experiment when they were ready to do so. The first 8 trials in the experiment were used for practice and instructional purposes and, therefore, were not included in any subsequent analyses. For half of the experiment ( 500 trials), the probability that the odd-colored target would repeat from one trial to the next was .80, whereas for the other half of the experiment (500 trials), this probability was set to 20 . Order of proportion-repeated condition was counterbalanced between participants, such that half of the participants performed the .80 block followed by the .20 block (.80- .20 group) and the other half performed the .20 block followed by the .80 block (.20-.80 group). There was no explicit cue or warning to participants that the probability of target repetitions would change at the halfway point of the experiment, nor were they informed about the proportionrepeated manipulation itself at any point before or during the experimental session. The first trial in each half of the experiment (the 1st and 501st) was randomly chosen as red or green with equal probability. The location of the odd-colored item in each display was determined randomly on each trial. The location of the gap in the odd-colored item (left or right) was also chosen randomly on each trial. An example of this procedure is shown in Fig. 1.

Following each experimental session, participants were asked to reflect on the first half of the experiment and to indicate their best estimate as to the percentage of trials on which the odd-colored target matched that on the previous trial. They were then asked to make the same judgment for the second half of the experiment. We recognize that this is not a particularly strong test of awareness of our proportion-repeated manipulation, but we did not want to draw participants' explicit attention to our manipulation by asking them to introspect after each proportion-repeated block. We therefore asked them to give proportion estimates only after all experimental trials were completed. Participants were then asked whether they had used any particular strategy to perform the search task, other than the explicit instructions given by the experimenter. If so, they were asked to briefly describe this strategy in writing. This question was intended to assess whether there was any deliberate and strategic use of the statistical structure of each block on the part of the participants. Following this 
Fig. 1 An example of the procedure used in Experiment 1. A target repetition is shown. Stimuli were presented on a black background, and stimuli appeared in green and red. Image is not to scale

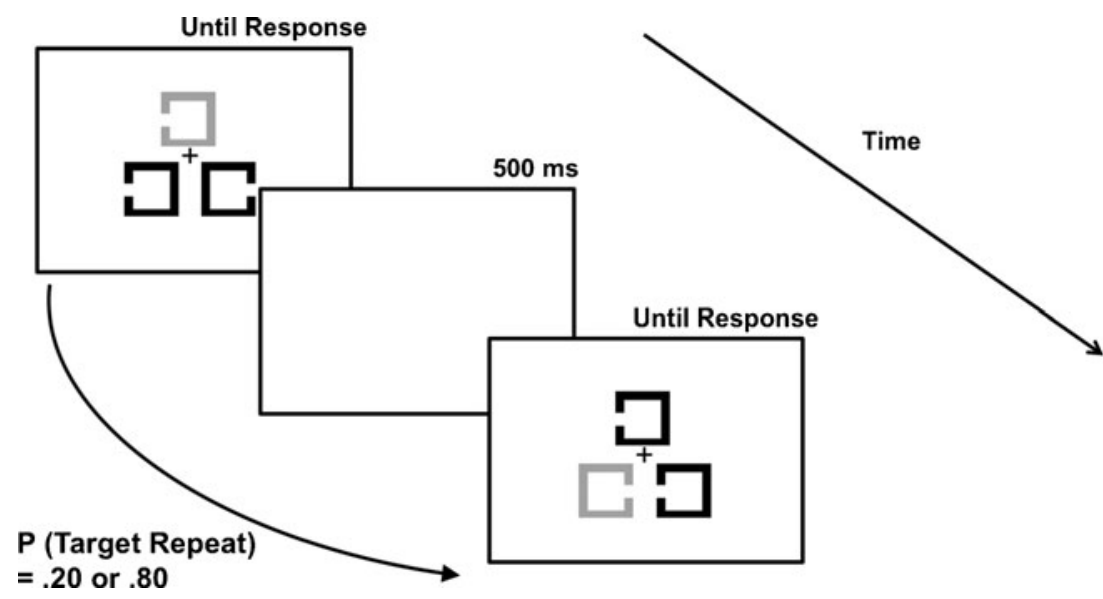

brief questionnaire, participants were debriefed on the nature of the task and given the opportunity to ask any questions.

Results

\section{Awareness data}

To assess whether participants were aware of the proportionrepeated manipulation within each experimental block, estimates of the proportion of target repeat trials for each block were submitted to one-sample $t$-tests that compared the mean proportion-repeated estimates with .5 , since a mean estimate of .5 would indicate that participants believed target repetitions and target switches to be equally represented within each block. Results of these comparisons showed that for the .80-.20 group, mean estimates were not significantly greater than .5 , in the .80 -proportion-repeated block $(M=.40, S D=.15)$ or significantly less than .5 in the .20 -proportion repeated block $(M=.48, S D=.20)$. Similarly, for the $.20-.80$ group, mean estimates were not significantly greater than .5 in the .80 -proportion-repeated block $(M=.56, S D=.14)$ or significantly less than .5 in the .20-proportion-repeated block $(M=.47, S D=.19)$. In addition, no participants indicated using a search strategy related to our manipulation. We therefore conclude that it is unlikely that participants made explicit use of our proportion-repeated manipulation to alter the way in which they performed the task.

\section{Response times}

First, we analyzed RTs as a function of proportion repeated (.80/.20), including block order as a between-subjects factor (.80-.20/.20-.80), to assess whether RTs are longer in the .20repeated condition, relative to the 80 -repeated condition, the result reported by Malkjovic and Nakayama (1994, Experiment 2). Only trials on which correct responses were made were submitted to this analysis. Correct RTs were first submitted to a nonrecursive outlier elimination procedure that removes observations on the basis of cell size (Van Selst \& Jolicoeur, 1994), in which $2.9 \%$ of the observations were removed. Results of the ANOVA revealed a significant main effect of proportion repeated, $F(1,22)=28.93, p<.001, \eta_{\mathrm{p}}^{2}=.57$, with responses being faster in the .80 -repeated block $(675 \mathrm{~ms})$ than in the .20 -repeated block ( $821 \mathrm{~ms})$. Interestingly, there was also a significant proportion-repeated $\times$ block order interaction, $F(1$, $22)=22.36, p<.001, \eta_{\mathrm{p}}^{2}=.50$. To examine this interaction further, follow-up paired $t$-tests were conducted. These comparisons revealed that for the .80-.20 group, RTs were similar in the .80 block $(717 \mathrm{~ms})$ and the .20 block $(735 \mathrm{~ms})$, whereas for the .20-.80 group, RTs were significantly shorter in the .80 block (633 ms) than in the .20 block $(907 \mathrm{~ms}), t(11)=5.16, p$ $<.001, d=1.49$. Overall, our results are generally consistent with those of Malkjovic and Nakayama (1994); however, there is an absence of slowing as the probability of a target switch trial increases for the .80-.20 group. This likely is owing to the fact that slowing due to a large proportion of target switch trials is additive with procedural learning, which generally speeds performance as experience with the task accumulates. Mean RTs as a function of proportion repeated and block order are depicted in Fig. 2.

Second, to address our primary question of interest, an ANOVA was conducted that treated target (repeat/switch) and proportion repeated (.80/.20) as within-subjects factors and block order (.80-.20/.20-.80) as a between-subjects factor. Since, for this analysis, each trial type is contingent on the nature of the previous trial type, only trials on which correct responses were made, as well as trials following correct responses, were included in the subsequent analysis. Consequently, only effects involving the target (repeat/switch) variable are reported. These mean RTs and corresponding error rates are shown in Fig. 3. Results revealed a significant main effect of target, with RTs being shorter overall for target repetitions $(686 \mathrm{~ms})$ than for switches $(818 \mathrm{~ms}), F(1,22)=$ $110.96, p<.001, \eta_{\mathrm{p}}^{2}=.84$; this is the PoP effect.

Most important, the results of the ANOVA revealed a significant target $\times$ proportion-repeated interaction, $F(1,22)$ 
Fig. 2 Mean correct response times are shown for each proportion-repeated block as a function of block order. Error bars represent corrected standard errors of the means (see Cousineau, 2005)

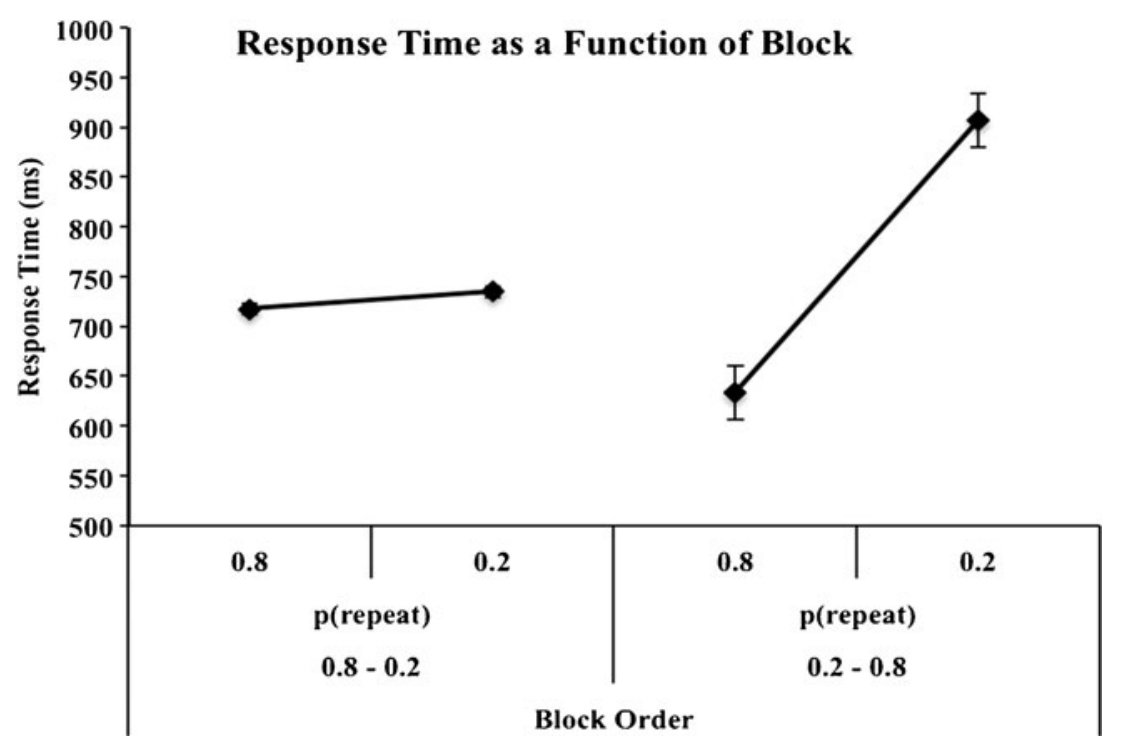

$=74.65, p<.001, \eta_{\mathrm{p}}^{2}=.72$, indicating that PoP effects did indeed differ as a function of the proportion of target repeat trials within each block. Interestingly, there was also a target $\times$ proportion-repeated $\times$ block order interaction, $F(1,22)=19.28$, $p<.001, \eta_{\mathrm{p}}^{2}=.47$. To examine the nature of this interaction further, separate 2 (target) $\times 2$ (proportion repeated) ANOVAs were conducted for the $.80-.20$ group and the $.20-.80$ group. Results for the $.80-.20$ group, revealed a significant target $\times$
Fig. 3 Mean response times for target repetitions and switches as a function of proportion-repeated and block order (top) and corresponding error rates (bottom). Error bars represent corrected standard errors of the means

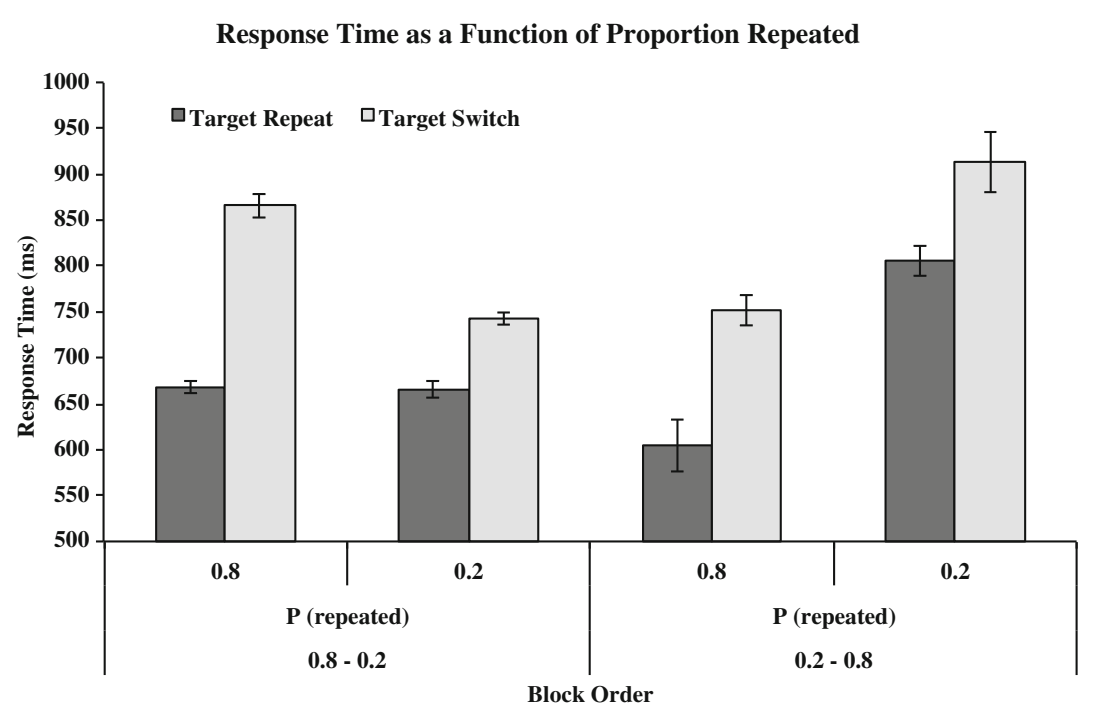

Error Rates as a Function of Proportion Repeated

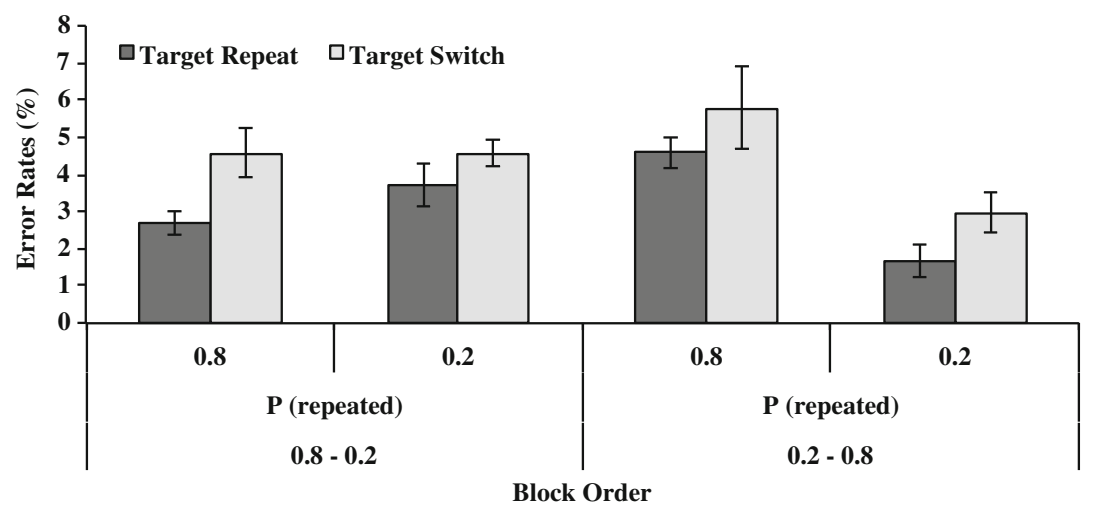


proportion-repeated interaction, $F(1,11)=161.06, p<.001$, $\eta_{\mathrm{p}}^{2}=.94$. Follow-up paired comparisons revealed significant PoP effects in both the .80 -repeated block $(197 \mathrm{~ms}), t(11)=$ $11.74, p<.001, d=3.39$, and the .20 -repeated block (77 ms), $t$ $(11)=6.57, p<.001, d=1.91$. Therefore, the target $\times$ proportion-repeated interaction is being driven by larger PoP effects in the .80-repeated block than in the .20-repeated block. Similarly, results for the $.20-.80$ group revealed a significant target $\times$ proportion-repeated interaction, $F(1,11)=6.13$, $p=.031, \eta_{\mathrm{p}}^{2}=.36$. Again, follow-up comparisons revealed significant PoP effects in both the .80 -repeated block $(147 \mathrm{~ms})$, $t(11)=8.77, p<.001, d=2.53$, and the .20 repeated block $(107 \mathrm{~ms}), t(11)=3.99, p=.002, d=1.15$. Taken together, the target $\times$ proportion-repeated $\times$ block order interaction observed in the omnibus ANOVA is driven by greater modulation in PoP effects across proportion-repeated blocks for the .80-.20 group, as compared with the $.20-.80$ group.

To assess the effects of our proportion-repeated manipulation in the absence of block order effects, we also opted to compare PoP effects in the .80 condition (but only for the participants who performed that condition first) with PoP effects in the.20 condition (but only for the participants who performed that condition first). In other words, RTs were entered into an ANOVA with target (repeat/switch) as a within-subjects factor and proportion repeated $(.80 / .20)$ as a between-subjects factor. Again, we observed a significant target $\times$ proportion-repeated interaction, $F(1,22)=7.94, p=.01, \eta_{\mathrm{p}}^{2}=.27$, indicating that PoP effects were larger for the .80-repeated group $(197 \mathrm{~ms})$ than for the .20-repeated group (108 ms).

We conducted an additional analysis to assess whether the modulation in PoP effects across proportion-repeated contexts would persist even with successive target color repetitions removed from the analysis. To that end, we analyzed target color repetitions and switches from trial $n-1$ to trial $n$, with the condition that trials $n-2$ and $n-1$ had different target colors. Geyer and Muller (2009) conducted a similar analysis in their study and called the contrast between target repetitions and switches first-order repetition effects. Notably, they found that first-order repetition effects were not affected by proportion repeated. To address this issue, first-order repetitions and switches were submitted to a repeated measures ANOVA that treated target and proportion-repeated block as within-subjects factors and block order as a between-subjects factor (note that this conditional analysis reduced cell sizes to 79 observations, on average, for the .20 condition and 79 and 20 observations for repeats and switches, respectively, in the .80 condition). Results showed a significant main effect of target, with responses being faster for first-order target repetitions $(699 \mathrm{~ms})$ than for switches $(812 \mathrm{~ms}), F(1,22)=107.11$, $p<.001, \eta_{\mathrm{p}}^{2}=.83$. In addition, there was a target $\times$ proportion-repeated interaction, $F(1,22)=22.26, p<.001$, $\eta_{\mathrm{p}}^{2}=.50$, as well as a target $\times$ proportion-repeated $\times$ block order interaction, $F(1,22)=5.70, p=.026, \eta_{\mathrm{p}}^{2}=.21$. To explain the interaction between target, proportion repeated, and block order, separate 2 (target) $\times 2$ (proportion repeated) ANOVAs were conducted for the $.80-.20$ group and the $.20-.80$ group. For the $.80-.20$ group, there was a significant main effect of target, $F(1,11)=75.39, p<.001, \eta_{\mathrm{p}}^{2}=.87$, with responses being faster on target repetitions $(687 \mathrm{~ms})$ than on switches $(802 \mathrm{~ms})$, as well as a target $\times$ proportion-repeated interaction, $F(1,11)=35.86, p<.001, \eta_{\mathrm{p}}^{2}=.77$. To explain this interaction, paired comparisons were conducted that revealed a significant PoP effect for the .80 -repeated block (155 ms), $t(11)=8.86, p<.001, d=2.56$, as well as for the .20 -repeated block (75 ms), $t(11)=6.49, p<.001, d=1.87$. For the $.20-.80$ group, there was a main effect of target, $F(1,11)=40.94, p<.001, \eta_{\mathrm{p}}^{2}=.61$, but no target $\times$ proportion-repeated interaction, with PoP effects being similar in the .80 -repeated block $(124 \mathrm{~ms})$ and the .20 -repeated block $(98 \mathrm{~ms})$. Overall, the analysis of first-order repetition effects shows that $\mathrm{PoP}$ is significantly larger in the .80 -repeated block than in the .20-repeated block, but only for the .80-.20 group.

Our analysis of first-order repetition effects described above was conducted to compare directly our work and that of Geyer and Muller (2009). However, an alternative means of controlling prior trial history was suggested to us by a reviewer, which we also opted to carry out. This procedure derives from the work of Jones and Sieck (2003) and, in the context of the present work, involves looking at priming effects in the following way: We computed mean RTs as a function of whether the target on trial $n$ was a repetition or alternation from trial $n-$ 1 and as a function of whether the target on trial $n-1$ was a repetition or alternation from trial $n-2$. This yields four trial types (repeat-repeat, repeat-switch, switch-repeat, switchswitch), allowing us to average repeat-repeat and switchrepeat trials together to get a measure of a target repetition from trial $n-1$ to trial $n$, with prior history controlled for, and to average repeat-switch and switch-switch trials together to get a measure of a target switch from trial $n-1$ to trial $n$, with prior trial history controlled for, while maintaining large cell sizes. We then entered these means into an ANOVA with target (repeat/switch) and proportion repeated (.80/.20) as withinsubjects factors and block order (.80-.20/.20-.80) as a between-subjects factor. We again observed a significant target $\times$ proportion-repeated $\times$ block order interaction, $F(1,22)=$ 5.07, $p=.035, \eta_{\mathrm{p}}^{2}=.19$. Again, to explain this interaction, separate 2 (target) $\times 2$ (proportion repeated) ANOVAs were conducted for the $.80-.20$ and $.20-.80$ block order groups.

For the $.80-.20$ group, there was a significant target $\times$ proportion-repeated block interaction, $F(1,11)=14.27$, $p=.003, \eta_{\mathrm{p}}^{2}=.57$. Follow-up paired comparisons revealed a significant PoP effect for the .80 -repeated block $(157 \mathrm{~ms}), t$ $(11)=7.48, p<.001, d=2.16$, as well as for the .20 -repeated block (94 ms), $t(11)=7.06, p<.001, d=2.04$. For the $.20-.80$ group, there was no target $\times$ proportion-repeated interaction, with PoP effects being similar in the .80 -repeated block 
(99 ms) and the .20-repeated block (115 ms). Overall, the analysis of priming effects (from trial $n-1$ to trial $n$ ) with prior trial history controlled for revealed the same pattern of results as our analysis of first-order repetition effects described above.

We conducted one final analysis to determine whether priming effects within each block change gradually as experience with our manipulation of proportion repeated accumulates. To address this issue, target repetitions and alternations were binned into two subblocks of 50 trials for the first 100 trials of each proportion-repeated condition and bins of 100 trials for the remaining trials within each proportion-repeated condition. We ran separate ANOVAs for $.80-.20$ and $.20-.80$ groups. For these analyses, target (repeat/switch), subblock $(1 / 2 / 3 / 4 / 5 / 6)$, and proportion repeated $(.80 / .20)$ were treated as within-subjects factors. Results of the analyses for the $.80-.20$ group, as well as the $.20-.80$ group, revealed that the target $\times$ proportionrepeated interaction did not interact with subblock, suggesting that the magnitude of the PoP effect measured on the first 50 trials did not change throughout the course of each proportion-repeated block. Mean RTs as a function of subblock are shown in Fig. 4.

\section{Error rates}

Mean error rates were submitted to an ANOVA that treated target (repeat/switch) and proportion repeated $(.80 / .20)$ as within-subjects factors and block order (.80-.20/.20-.80) as a between-subjects factor. Results of this analysis revealed a significant main effect of target, $F(1,22)=7.42, p=.012$, $\eta_{\mathrm{p}}^{2}=.25$, with fewer errors committed on target repetitions $(3.19 \%)$ than on switches $(4.46 \%)$. There was also a significant proportion-repeated $\times$ block order interaction, $F$ $(1,22)=9.45, p=.006, \eta_{\mathrm{p}}^{2}=.30$. Follow-up paired $t$-tests revealed that for the $.80-.20$ group, error rates were similar in the $.80-(3.6 \%)$ and $.20-(4.1 \%)$ repeated conditions, $t<1$, whereas for the $.20-.80$ group, error rates were lower in the .20 -repeated condition $(2.3 \%)$ than in the .80 -repeated condition $(5.2 \%), t(11)=2.98, p=.012, d=0.86$. Overall, the pattern of error rates does not favor a speed-accuracy trade-off interpretation of the pattern of RTs.

\section{Discussion}

The primary purpose of Experiment 1 was to assess whether PoP effects are subject to some degree of modulation when the probability of a target repeat trial is high (.80) versus low (.20). We found that PoP effects in the .80 block were significantly larger than those in the .20 block. This result is to be expected, since mean target repeat RTs in the .80 condition derive from large runs of same-color target trials, which are known to produce cumulative benefits to performance and which must, therefore, increase the resulting priming effects. Crucially, however, when prior trial history was controlled for, a significant modulation of PoP as a function of proportion-repeated block remained. This result may owe to the fact that our comparison of priming effects between a .20-repeated and .80 -repeated block provided a much stronger contrast than the comparison conducted by Geyer and Muller (2009). In addition, first-order effects were computed among a sample of 12 unpracticed observers in the present study, whereas there were 8 in Geyer and Muller. It may, therefore, be the case that participants performed the search task in functionally equivalent ways in the present study and in that of Geyer and Muller but that first-order effects were simply too weak to be measured in the Geyer and Muller study.

It was somewhat surprising to us that PoP effects did not change more gradually as observers gained more experience in each block but, rather, remained relatively stable beyond the first 50 trials. However, context-specific proportionrepeated effects have been shown to emerge relatively quickly in other domains, such as Stroop (Crump, Gong, \& Milliken, 2006; Jacoby, Lindsay, \& Hessels, 2003; see also Crump \& Milliken, 2009). Nonetheless, one might expect that learning would unfold more slowly in the second block of trials after observers have already been biased by the previous block. While any explanation of the fast emergence of PoP in-line with proportion repeated is post hoc and admittedly speculative, it could be that in the second block of trials, the contrast between current and prior probability of repetition is more salient and so one's "expectations" quickly readjust. The rapid nature of the learning observed here will be an interesting issue for future study.

Interestingly, we observed a strong influence of block order on the PoP $\times$ proportion-repeated interaction, which suggests that the experiences one has with the structure inherent in the task early on carries forward to impact search performance throughout the experiment. In addition to the presence of significant modulation of PoP across blocks for first-order repetition effects, the effects of block order, while not explicitly predicted, converge on the idea that the effects of proportion-repeated manipulations on PoP likely result from a form of learning. We sought additional evidence for this claim in Experiment 2.

\section{Experiment 2}

The primary goal of Experiment 2 was to replicate the pattern of results obtained in Experiment 1, while controlling for prior trial history (preceding $n-1$ ) that may modulate PoP effects in the absence of any learning or expectancy. Specifically, performance has been shown to improve steadily as the number of prior target repeat trials increases (cumulative repetition benefits; Malkjovic \& Nakayama, 1994). In light of this, 
Fig. 4 Mean response times for target repetitions and switches as a function of proportion repeated and subblock (trial bin), for the .80-.20 group (top) and the .20-.80 group (bottom). Error bars represent corrected standard errors of the means

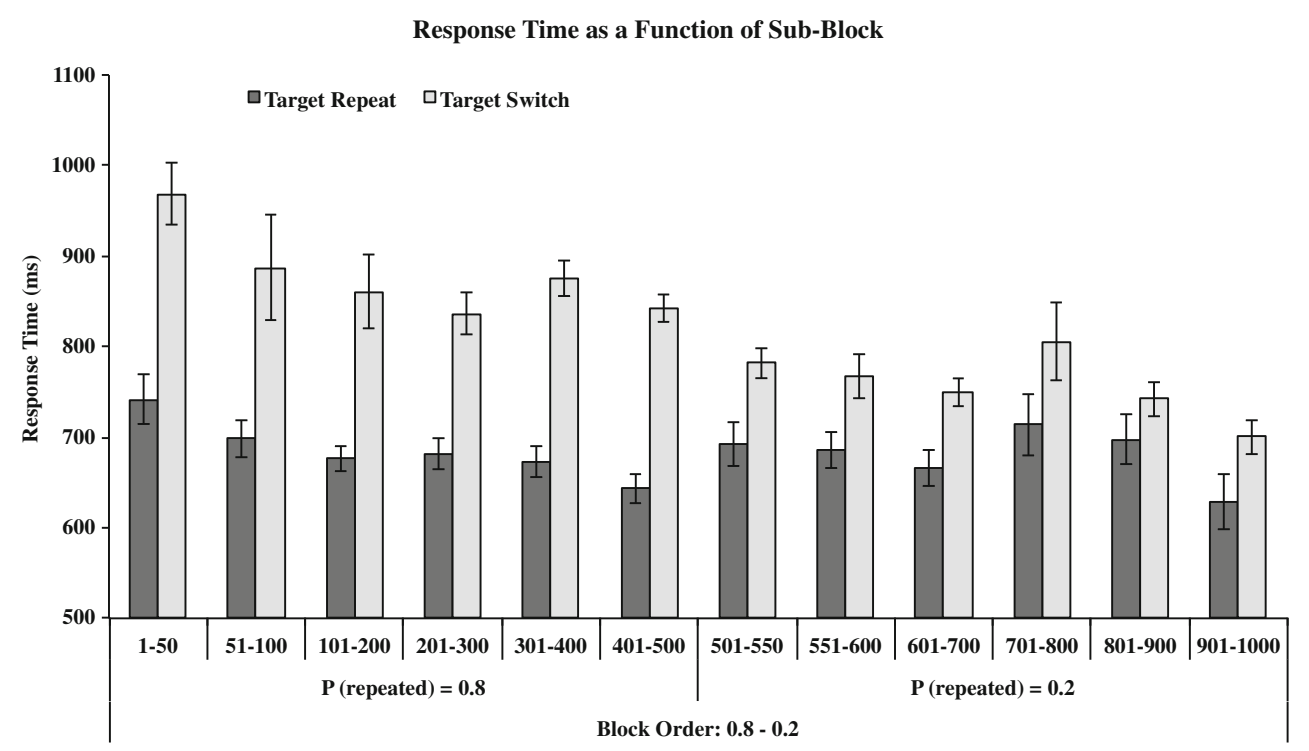

Response Time as a Function of Sub-Block

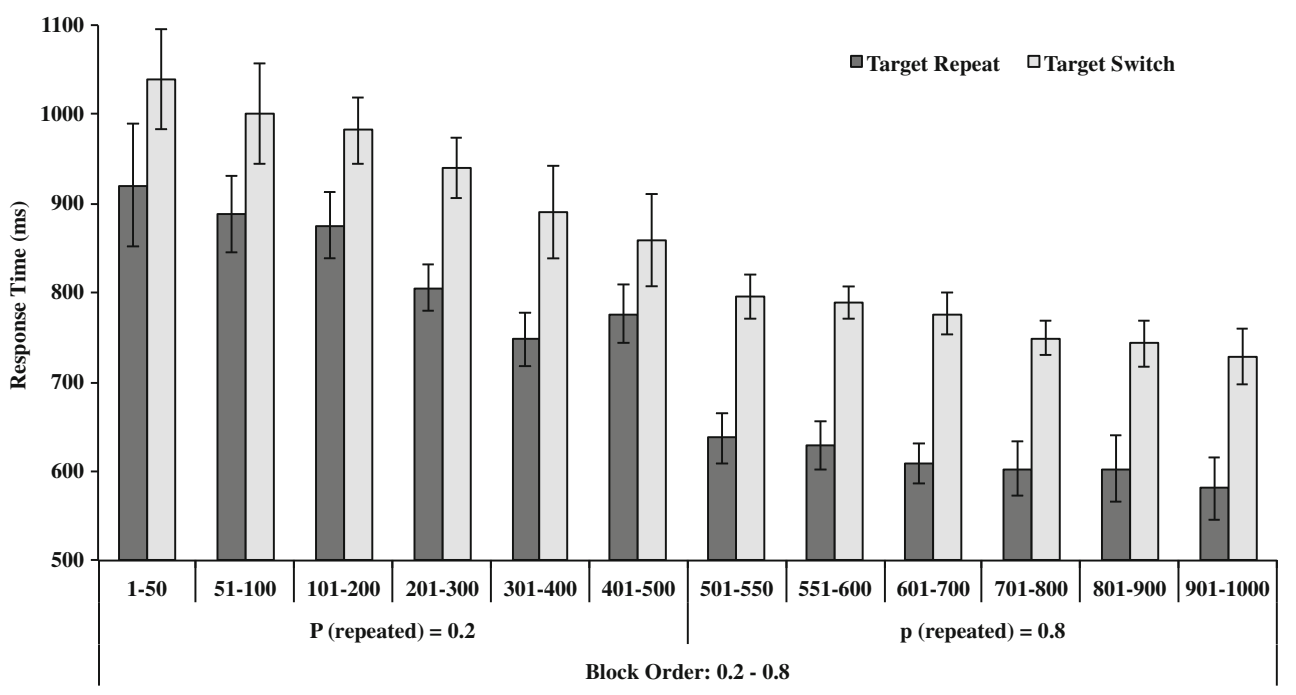

Experiment 2 was designed such that the probability of successive repetitions of target color contributing to the mean target repeat RTs was similar in both the high- and lowproportion-repeated blocks.

Recall that Geyer and Muller (2009) were unable to measure differences in first-order repetition effects between experiments with a high proportion of target repeat trials and a baseline experiment. In their analysis, only target repeat trials following alternations were analyzed, so as to remove the effects of cumulative repetitions. This strategy may have actually produced an additional difficulty in measuring firstorder repetition differences, however. Consider the highproportion-repeated condition of Experiment 1 here and Experiment 3 in Geyer and Muller. If the relationship between trial $n-1$ and trial $n$ is analyzed with the condition that the target on trial $n-1$ differs from that on trial $n-2$ (as in an analysis of first-order effects), then it stands to reason that there would be large strings of target repeat trials preceding trial $n-2$ that differ in identity from trial $n$ (since there is a high probability of target repetitions in these experiments). Given that performance on trial $n$ is influenced by the target identity not only on trial $n-1$, but also on prior trials (Malkjovic \& Nakayama, 1994), this prior trial history may act to reduce priming effects measured on trial $n$, which may, in turn, counteract any top-down control afforded by the predictability inherent in the experiment. As a result, repetition effects from trial $n-1$ to trial $n$ must be assessed between proportion-repeated blocks, with prior trial history equated between the two blocks. The analytic procedure based on Jones and Sieck (2003) offered one way to address this issue in Experiment 1, whereas a subtle change to the method was introduced to address this issue in Experiment 2. If differences in PoP as a function of proportion repeated persist, it may be argued that trial-to- 
trial priming effects are affected by the predictability of trialto-trial contingencies in a context-specific manner.

\section{Method}

\section{Participants}

Twenty-two undergraduates at McMaster University (8 male, 14 female; $M_{\text {age }}=18.3$ years) enrolled in introductory psychology took part in exchange for course credit. All participants had normal or corrected-to-normal vision.

\section{Apparatus and stimuli}

The apparatus and stimuli were identical to those used in Experiment 1.

\section{Procedure}

The procedure was similar to that in Experiment 1, with the following exceptions: The response-to-stimulus interval (RSI) was varied between trials on a predictable basis, such that every second trial was preceded by a $500-\mathrm{ms}$ blank interval and every other trial was preceded by a $4,000-\mathrm{ms}$ blank interval. Our manipulation of the proportion of target repetitions in each block (either .80 or .20) was applied only to the trial pairs that were separated by a 500-ms RSI, whereas the probability of a target repeat for trial pairs separated by the 4,000-ms RSI was held at .50 (chance). In this way, only trials following a 500-ms RSI were included in the primary analysis of RT, since those were the only trials on which proportion repeated was manipulated. This procedure implies that regardless of whether one is in the high- or low-proportion-repeated block, given that the current trial is a target repetition relative to trial $n-1$, the probability that trial $n-2$ contains the same target color is .5.

One implication of this procedure is that it might well be difficult to observe proportion-repeated influences on target repetition effects, because only half of the trial pairs in each block have a high or low probability of being repetitions. In other words, by applying our proportion-repeated manipulation to only half of the trials in the experiment, the actual probabilities of receiving a target repeat trial in the .80- and .20 -repeated blocks were .65 and .35 , respectively. It is for this reason that we chose to associate the .80- and .20repeated trial pairs with a different RSI $(500 \mathrm{~ms})$ than the .50 -repeated trial pairs $(4,000-\mathrm{ms} \mathrm{RSI})$. We reasoned that by distinguishing these different trial types temporally, learning may occur for one trial-pair relationship, independent of any learning (or lack thereof) that would occur with the other trial-pair relationship. This logic is partially motivated by prior work demonstrating that priming effects can arise independently for different trial contexts (see Thomson \&
Milliken, 2012, in press). An example of this procedure is shown in Fig. 5.

As in Experiment 1, order of proportion-repeated condition was counterbalanced between participants, and at the end of the experimental session, participants were asked to make explicit estimates of the proportion of target repeat trials within each half of the experiment. Here, however, participants were asked to restrict their estimates only to pairs of trials that occurred close together in time-that is, only the trials on which the probability of a target repetition was manipulated.

Results

\section{Awareness data}

As in Experiment 1, estimates of the proportion of target repeat trials for each block were submitted to one-sample $t$-tests that compared the mean proportion-repeated estimates with .5 (chance). Results of these comparisons showed that for the group that performed the .80-proportion-repeated block first, mean estimates were not significantly greater than .5 in the .80 proportion-repeated block $(M=.52, S D=.18)$ or significantly less than .5 in the .20 -proportion-repeated block $(M=.51$, $S D=.10)$. Similarly, for the group that performed the .20 proportion-repeated block first, mean estimates were not significantly greater than .5 in the .80 -proportion-repeated block $(M=.51, S D=.21)$ or significantly less than .5 in the .20 proportion-repeated block $(M=.53, S D=.25)$. In addition, no participants indicated using a search strategy related to our manipulation. We therefore conclude that it is unlikely that participants made explicit use of our proportion-repeated manipulation to alter the way in which they performed the task.

\section{Response times}

First, we conducted an ANOVA that treated proportion repeated $(.80 / .20)$ as a within-subjects factor and block order as a between-subjects factor. As in Experiment 1, correct responses were first submitted to an outlier elimination procedure that removed $3.0 \%$ of observations from further analysis. Mean RTs for the remaining observations were subsequently submitted to the analysis. The analysis revealed a main effect of proportion repeated that approached significance, $F(1,20)=$ $3.80, p=.065, \eta_{\mathrm{p}}^{2}=.16$, with a tendency for shorter RTs in the .80 -repeated block $(700 \mathrm{~ms})$ than in the .20 -repeated block (762 ms). The proportion-repeated $\times$ block order interaction also failed to reach significance, $F(1,20)=3.93, p=.061$, $\eta_{\mathrm{p}}^{2}=.16$, but again this effect reflected the same qualitative pattern of RTs as that observed in Experiment 1.

Second, to address our primary question of interest, an ANOVA was conducted that treated target (repeat/switch) and proportion repeated $(.80 / .20)$ as within-subjects factors 
Fig. 5 An example of the procedure used in Experiment 2. Manipulation of proportion of target repeat trials was applied only to trials separated by a 500 -ms response-tostimulus interval (RSI; first and third panels), whereas no proportion-repeated manipulation was applied to trials separated by a $4,000-\mathrm{ms}$ RSI (third and fifth panels). Stimuli were presented on a black background, and stimuli appeared in green and red. Image not to scale

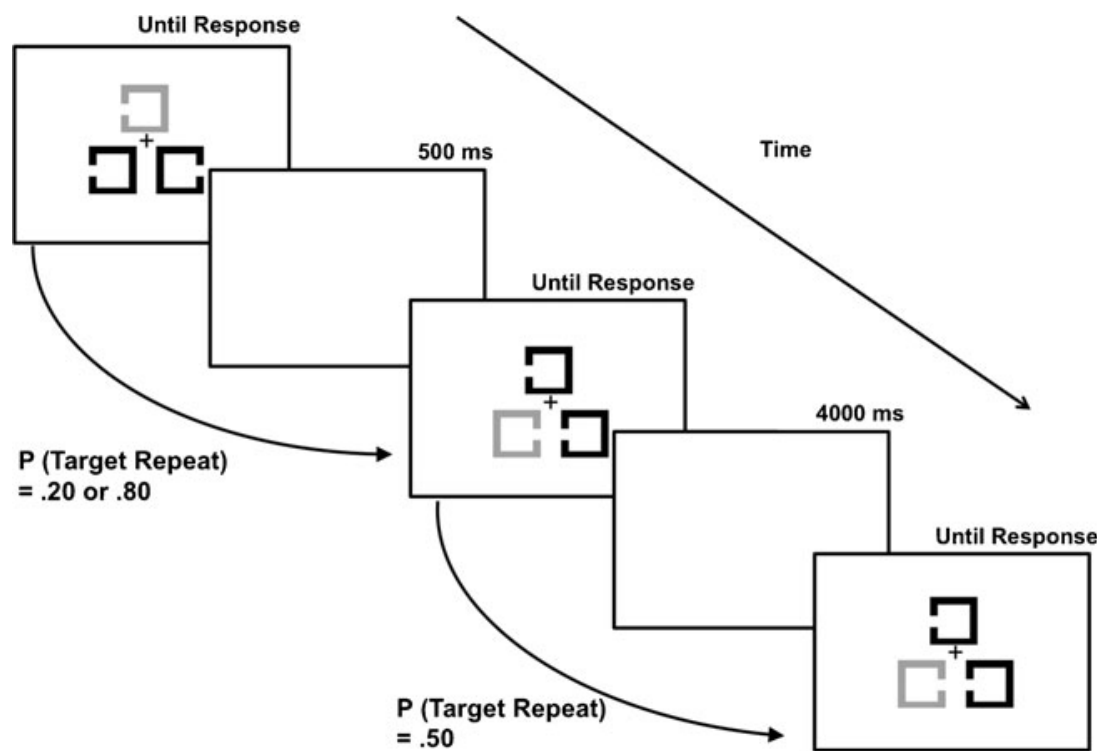

and block order $(.80-.20 / .20-.80)$ as a between-subjects factor. As in Experiment 1, only trials on which correct responses were made, as well as trials following correct responses, were included. Results of this analysis revealed a significant main effect of target, with RTs being shorter, overall, for target repetitions (666 ms) than for switches (797 ms), $F(1,20)=81.97, p<.001, \eta_{\mathrm{p}}^{2}=.80$ (the PoP effect).

Most important, the results of the ANOVA revealed a significant target $\times$ proportion-repeated interaction, $F(1,20)=16.05, p=.001, \eta_{\mathrm{p}}^{2}=.45$. Interestingly, there was also a target $\times$ proportion-repeated $\times$ block order interaction, $F(1,20)=7.23, p=.014, \eta_{\mathrm{p}}^{2}=.27$. To examine the nature of this interaction, separate 2 (target) $\times 2$ (proportion repeated) ANOVAs were conducted for the .80-.20 group and the $.20-.80$ group. For the $.80-.20$ group, results revealed a significant target $\times$ proportion-repeated interaction, $F(1,10)=$ 15.62, $p=.003, \eta_{\mathrm{p}}^{2}=.61$. Follow-up paired comparisons revealed significant PoP effects in both the .80 -repeated block (212 ms), $t(10)=6.96, p<.001, d=2.10$, and in the .20 repeated block (139 ms), $t(10)=5.39, p<.001, d=1.63$. For the $.20-.80$ group, results revealed no significant target $\times$ proportion-repeated interaction, with priming effects being similar in the .80-repeated block (94 ms) and .20-repeated block $(80 \mathrm{~ms})$. Taken together, the target $\times$ proportionrepeated $\times$ block order interaction observed in the omnibus ANOVA is driven by modulation in PoP effects across proportion-repeated blocks for the $.80-.20$ group but no significant modulation for the $.20-.80$ group.

Again, to assess the effects of our proportion-repeated manipulation in the absence of block order effects, we compared PoP effects in the .80 condition for the participants who performed that condition first with PoP effects in the. 20 condition for the participants who performed that condition first. To that end, RTs were entered into an ANOVA with target (repeat/switch) as a within-subjects factor and proportion repeated $(.80 / .20)$ as a between-subjects factor. Again, we observed a significant target $\times$ proportion-repeated interaction, $F$ $(1,20)=15.41, p=.001, \eta_{\mathrm{p}}^{2}=.44$, indicating that PoP effects were larger for the .80 -repeated group $(197 \mathrm{~ms})$ than for the .20-repeated group $(108 \mathrm{~ms})$. RTs and corresponding error rates are shown in Fig. 6.

As a final analysis, we looked at priming effects across the 4,000-ms transitions (for which the probability of receiving a target repetition was .5) to examine whether the modulation observed for the .80- and .20-repeated trials carried over to the other half of the trials or whether this modulation was confined only to the trials for which there was a proportion-repeated manipulation. In this way, we can assess how general or specific the learning was. To that end, an ANOVA was conducted that treated target (repeat/switch) and proportion-repeated block $(.80 / .20)$ as within-subjects factors and block order (.80-.20/ $.20-80)$ as a between-subjects factor. Importantly, there was a significant target $\times$ proportion-repeated interaction, $F(1,20)=$ 15.51, $p=.001, \eta_{\mathrm{p}}^{2}=.44$. This interaction was driven by significant $\mathrm{PoP}$ in the .80 proportion-repeated block $(65 \mathrm{~ms}), t$ $(21)=4.65, p<.001, d=0.99$, and no significant $\mathrm{PoP}$ in the .20 proportion-repeated block $(12 \mathrm{~ms})$. This result suggests that the modulation of $\mathrm{PoP}$ as a function of proportion repeated in this experiment was quite general and not confined to the trials on which proportion repeated was manipulated (i.e., trials separated by $500 \mathrm{~ms})$.

\section{Error rates}

Mean error rates were submitted to an ANOVA that treated target (repeat/switch) and proportion repeated (.80/.20) as within-subjects factors, and block order (.80-.20/.20-.80 first) as a between-subjects factor. Results of this analysis revealed 
Fig. 6 Mean response times for target repetitions and switches as a function of proportion repeated and block order (top) and corresponding error rates (bottom). Error bars represent corrected standard errors of the means
Response Time as a Function of Proportion Repeated

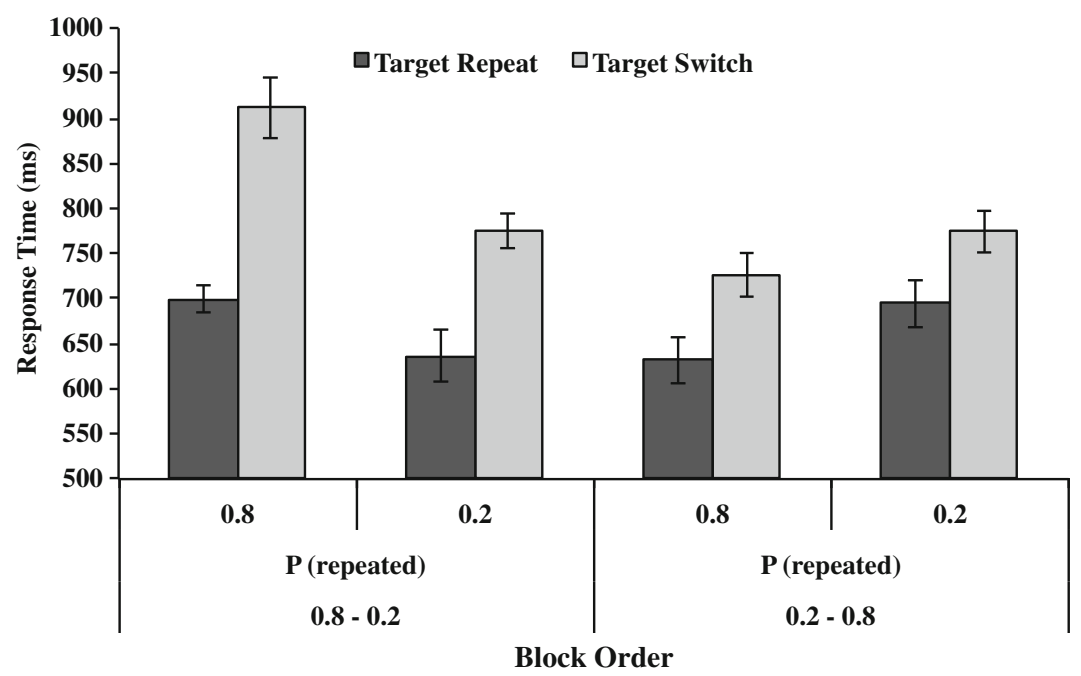

Error Rates as a Function of Proportion Repeated

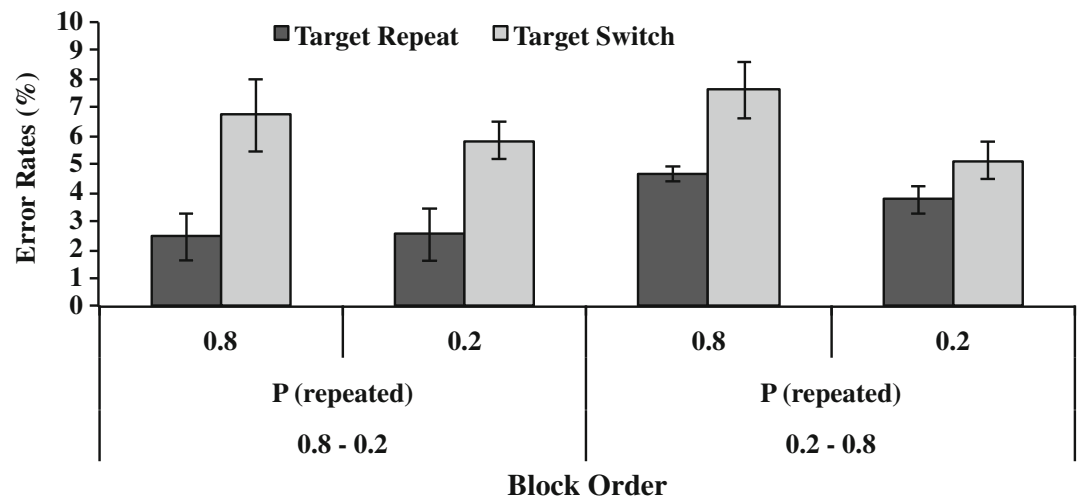

a significant main effect of target, $F(1,20)=13.83, p=.001$, $\eta_{\mathrm{p}}^{2}=.41$, with fewer errors committed on target repetitions $(3.4 \%)$ than on switches $(6.3 \%)$. No other effects reached significance in the analysis of error rates. Overall, the pattern of error rates does not favor a speed-accuracy trade-off interpretation of the pattern of RTs.

\section{Discussion}

The purpose of Experiment 2 was to assess whether PoP effects would vary as a function of proportion repeated with prior trial history controlled for. Despite this control for cumulative repetition effects, the same qualitative pattern of results emerged as in Experiment 1. PoP effects were significantly smaller in a low-proportion-repeated condition than in a high-proportion-repeated condition. Once again, this effect interacted with block order, but this time, significant modulation in PoP was observed only for the participants who performed the high-proportion-repeated block first, a point we will return to in the General discussion section.

\section{General discussion}

The primary purpose of the work reported here was to assess whether intertrial priming effects in singleton search can be modulated in accord with the statistical structure inherent within a block of trials. In Experiment 1, it was shown that, in general, mean RTs in a color pop-out search task increase as a function of the number of target color switch trials within a block - a replication of the results of Malkjovic and Nakayama (1994). Additionally, we examined trial-to-trial priming effects as a function of proportion repeated to assess whether such effects can be modulated in-line with one's expectation for a target repetition or alternation, similar to the work of Geyer and Muller (2009). The effects of proportion repeated on PoP seen here extend and build upon the work of Geyer and Muller, who presented evidence that cumulative repetition benefits are larger in an experimental context with a high proportion of target color repetitions, as compared with a baseline context. Here, we show that trial-totrial repetition effects in color pop-out search are also strongly affected by the proportion of target color repetitions within a 
search context, even when cumulative repetitions of target color are controlled for. In addition, we show that this modulation appears to occur in the absence of any strategic intervention on the part of the observer. Finally, the modulation of PoP effects as a function of proportion repeated emerges early on (within 50 trials) and seems to depend heavily on the nature of one's prior experience with the task in the long term (as evidenced through strong effects of block order, which we shall return to shortly). As such, the empirical work reported here is supportive of the early findings of Hillstrom (2000) and constitutes strong evidence for the role of expectancy in modulating repetition effects in singleton search that are not owing to successive benefits.

The strong effect of block order on the modulation of PoP across proportion-repeated blocks was somewhat unexpected and deserves further comment. While we have interpreted the effects of block order as evidence of learning (in the longer term), it is not entirely clear why PoP effects should be more greatly modulated when the high-proportion-repeated block is performed first, relative to second. At this point we can only speculate on this issue, but it seems possible that a contingency based on feature mismatches from one experience to another may be harder to learn than a contingency based on feature matches (see Fiacconi \& Milliken, 2012; Vaquero, Fiacconi, \& Milliken, 2010). Another possibility is that participants' experiences with naturalistic regularities in the environment affect their ability to learn about alternations in our contrived experimental procedures. In particular, in the real world, one may encounter any number of different stimuli at any given point in time. Consequently, the absence of one particular stimulus does not imply the presence of another particular stimulus. When we introduce this particular contingency in an experiment, therefore, it may not be surprising that probabilistic alternations remain unnoticed. Again, this is merely speculation at this point and is an issue for further study.

We have argued that PoP effects are subject to a form of attentional control that is related on ones expectations about likely target colors but have, thus far, remained fairly agnostic with respect to the specific mechanisms by which this control is exerted. Any mechanistic explanation of the observed modulation of PoP as a function of proportion repeated will be necessarily dependent on the theoretical locus of PoP effects one subscribes to. For instance, if PoP effects derive from adjustments in "weights" or "gains" to abstract feature representations on a trial-by-trial basis, as some have argued (Lee, Mozer, \& Vecera, 2009; Malkjovic \& Nakayama, 1994, 1996; Maljkovic \& Nakayama, 2000), then attentional control processes may mediate the amount of weight given to target colors on the basis of the probability that those colors will be encountered again on a subsequent trial. This type of control may serve to adjust the contribution of target feature representations to an overall salience map that guides pop-out search (as per Geyer \& Muller, 2009; Wolfe, 1994; Wolfe et al., 2003).
Another theoretical account of the locus of PoP effects is one in which priming effects result from the retrieval of bound memory representations in an implicit and automatic manner on each trial, similar to instances (Logan, 1988, 1990) or event-files (Hommel, 1998, 2004). If PoP is driven by the recruitment of a memory episode created on the previous trial, as some have suggested (Hillstrom, 2000; Huang, Holcombe, \& Pashler, 2004; Thomson \& Milliken, 2011, in press), then the modulation in PoP effects seen here is likely driven by attentional processes that control the extent to which a retrieved episode should be integrated with current performance. Such control would act to allow fluid integration in a context in which target colors likely repeat from one trial to the next (thus increasing resulting priming effects) and to restrict integration in a context in which target colors likely mismatch from one trial to the next (thus decreasing the resulting priming effects).

Future work will certainly be necessary to determine whether attentional control operates on intertrial priming effects in singleton search in a feed-forward manner (by adjusting feature weights in accord with environmental statistics), in a retrospective manner (by affecting the degree of integration of a past experience with current perception), or both. Recent work from our lab however, has shown that the number of trials over which PoP can be measured is strongly affected by contextual reinstatement that is both perceptual (Thomson \& Milliken, 2012) and nonperceptual (Thomson \& Milliken, in press) in nature, suggesting that retrieval interference may at least partially dictate the magnitude of PoP effects. In addition, recent theoretical accounts of PoP have been forwarded that acknowledge a role for both feed-forward mechanisms, as well as episodic retrieval contributions to the PoP effect (Lamy, Yashar, \& Ruderman, 2010; Yashar \& Lamy, 2011). As a result, the attentional control over the magnitude of PoP shown in the present work could result from either control over feature weights from one trial to the next or control over event integration, if indeed both such processes contribute to PoP effects. It is worth mentioning, however, that none of the feed-forward, retrospective, or hybrid accounts of PoP effects can fully account for the strong effects of block order observed across both experiments. Nevertheless, manipulations of the proportion of target repetitions within a block of trials may serve as a useful empirical tool in future work to better delineate the locus of PoP effects.

Finally, an important goal of future work on attentional control over PoP effects will be to determine when exactly such control is exerted during a search trial. For instance, if control is implemented at the level of feature weights that sum to a master salience map, those weights will be set on one trial and will carry forward to a subsequent search trial. In this way, top-down control over feature weights would serve to bias the local salience signal corresponding to the pop-out target and may actually affect the time required for focal attention to be 
drawn to the singleton. That is, such control may impact the search process itself. In contrast, if control over PoP effects is implemented by controlling the degree of episodic integration between a current and prior search trial, it is likely that the match or mismatch between current and prior experience will serve to affect some "decision" stage (see Huang et al., 2000) about whether or not the singleton is indeed the target. This account implies that attentional control over PoP effects may actually affect not the search process but, rather, processing that occurs later in time.

In summary, the work presented here constitutes strong support for the view that intertrial priming effects in singleton search are affected by the expectations, or attentional "set," of the observer (see also Fecteau, 2007, who showed that target features need to be part of an active task "set" in order to produce priming effects). Furthermore, these effects of "expectation" over the magnitude of PoP that occur in-line with the statistical structure of the task are not driven by cumulative repetitions that are more prevalent in a high-proportionrepeated block. Thus, PoP seems to be affected not only by the immediately preceding trials, but also by longer-term learning within each block, and even by learning that took place in earlier blocks (for evidence that PoP operates across multiple time scales, see also Brascamp, Pels, \& Kristjansson, 2011; Martini, 2010). Although we have shown that PoP can be modulated by the likelihood of target repetitions, our results also fit with those of Malkjovic and Nakayama (1994), who showed that such manipulations cannot overcome PoP effects, no matter how beneficial it would be to performance. In this way, the striking automaticity of PoP effects is also emphasized in the work presented here, since robust PoP was observed in the low-proportion-repeated conditions across both experiments. Nevertheless, the influence of the memory representation for a prior trial on current performance in a simple color pop-out search task is not a constant but is, instead, sensitive to the statistical structure inherent in an observer's environment.

Acknowledgment This work was funded by an NSERC Discovery Grant awarded to B.M. In addition, we would like to thank Michael C. Mozer as well as two anonymous reviewers for their insightful comments and suggestions on an earlier version of the manuscript. We also thank Maria C. D'Angelo.

\section{References}

Brascamp, J. W., Pels, E., \& Kristjansson, A. (2011). Priming of pop-out on multiple time scales during visual search. Vision Research, 51 (17), 1972-1978.

Bravo, M., \& Nakayama, K. (1992). The role of attention in different visual search tasks. Perception \& Psychophysics, 51, 465-472.

Cousineau, D. (2005). Confidence intervals in within-subjects designs: A simpler solution to Loftus and Masson's method. Tutorials in Quantitative Methods for Psychology, 1, 42-45.
Crump, M. J. C., Gong, Z., \& Milliken, B. (2006). The context-specific proportion congruent Stroop effect: Location as a contextual cue. Psychonomic Bulletin \& Review, 13, 316-321.

Crump, M. J. C., \& Milliken, B. (2009). The flexibility of context-specific control: Evidence for context-driven generalization of item-specific control settings. The Quarterly Journal of Experimental Psychology, 62, 1523-1532.

Fecteau, J. H. (2007). Priming of pop-out depends upon the current goals of observers. Journal of Vision, 7, 1-11.

Fiacconi, C. M., \& Milliken, B. (2012). Contingency blindness: Location-identity binding mismatches obscure awareness of spatial contingencies and produce profound interference in visual working memory. Memory \& Cognition. Advance online publication. doi:10.3758/s13421-012-0193-5

Geyer, T., \& Muller, H. J. (2009). Distinct, but top-down modulable color and positional priming mechanisms in visual pop-out search. Psychological Research, 73, 167-176.

Hillstrom, A. P. (2000). Repetition effects in visual search. Perception \& Psychophysics, 62, 800-817.

Huang, L., Holcombe, A. O., \& Pashler, H. (2004). Repetition priming in visual search: Episodic retrieval, not feature priming. Memory \& Cognition, 32, 12-20.

Hommel, B. (1998). Event files: Evidence for automatic integration of stimulus-response episodes. Visual Cognition, 5, 183-216.

Hommel, B. (2004). Event files: Feature binding in and across perception and action. Trends in Cognitive Sciences, 8, 494-500.

Jacoby, L. L., Lindsay, D. S., \& Hessels, S. (2003). Item-specific control of automatic processes: Stroop process dissociations. Psychonomic Bulletin \& Review, 10, 638-644.

Jones, M., \& Sieck, W. R. (2003). Learning myopia: An adaptive recency effect in category learning. Journal of Experimental Psychology: Learning, Memory, and Cognition, 29, 626-640.

Lamy, D., Yashar, A., \& Ruderman, L. (2010). A dual-stage account of inter-trial priming effects. Vision Research, 50, 1396-1401.

Lee, H., Mozer, M. C., \& Vecera, S. P. (2009). Mechanisms of priming of pop-out: Stored representations or feature-gain modulations? Attention, Perception, \& Psychophysics, 71, 1059-1071.

Logan, G. D. (1988). Toward an instance theory of automatization. Psychological Review, 95, 492-527.

Logan, G. D. (1990). Repetition priming and automaticity: Common underlying mechanisms? Cognitive Psychology, 22, 1-35.

Malkjovic, V., \& Nakayama, K. (1994). Priming of pop-out: I. Role of features. Memory \& Cognition, 22, 657-672.

Malkjovic, V., \& Nakayama, K. (1996). Priming of pop-out: II. The role of position. Perception \& Psychophysics, 58, 977-991.

Maljkovic, V., \& Nakayama, K. (2000). Priming of pop-out: III. A short-term implicit memory system beneficial for rapid target selection. Visual Cognition, 7, 571-595.

Martini, P. (2010). System identification in priming of pop-out. Vision Research, 50, 2110-2115.

Nissen, M. J., \& Bullemer, P. (1987). Attentional requirements of learning: Evidence from performance measures. Cognitive Psychology, 19, 1-32.

Thomson, D. R., \& Milliken, B. (2011). A switch in task affects priming of pop-out: Evidence for the role of episodes. Attention, Perception, \& Psychophysics, 73, 318-333.

Thomson, D. R., \& Milliken, B. (2012). Perceptual distinctiveness produces long-lasting priming of pop-out. Psychonomic Bulletin \& Review, 19, 170-176.

Thomson, D. R., \& Milliken, B. (in press). Contextual distinctiveness produces long-lasting priming of pop-out. Journal of Experimental Psychology: Human Perception and Performance. Advance online publication. doi:10.1037/a0028069

Van Selst, M., \& Jolicoeur, P. (1994). A solution to the effect of sample size on outlier elimination. The Quarterly Journal of Experimental Psychology, 47, 631-650. 
Vaquero, J. M. M., Fiacconi, C. M., \& Milliken, B. (2010). Attention, awareness of contingencies, and control in spatial localization: A qualitative difference approach. Journal of Experimental Psychology. Human Perception and Performance, 36, 1342 1357.

Wolfe, J. M. (1994). Guided search 2.0 A revised model of visual search. Psychonomic Bulletin \& Review, 1, 202-238.
Wolfe, J. M., Butcher, S. J., Lee, C., \& Hyle, M. (2003). Changing your mind: On the contributions of top-down and bottom-up guidance in visual search for feature singletons. Journal of Experimental Psychology. Human Perception and Performance, 29, 483-502.

Yashar, A., \& Lamy, D. (2011). Refining the dual-stage account of intertrial feature priming: Does motor response or response feature matter? Attention, Perception, \& Psychophysics, 73, 2160-2167. 